\title{
Wentzel-Bardeen singularity and phase diagram for interacting electrons coupled to acoustic phonons in one dimension
}

\author{
Daniel Loss ${ }^{1}$ and Thierry Martin ${ }^{2 *}$ \\ 1 Department of Physics, Simon Fraser University, Burnaby, BC, Canada V5A1S6 \\ 2 Theoretical Division, CNLS, Los Alamos National Laboratory, Los Alamos, NM 87545
}

\begin{abstract}
We consider strongly correlated electrons coupled to low energy acoustic phonons in one dimension. Using a Luttinger liquid description we calculate the exponents of various response functions and discuss their striking sensitivity to the Wentzel-Bardeen singularity induced by the presence of phonons. For the Hubbard model plus phonons the equivalent of a phase diagram is established. By increasing the filling factor towards half filling the Wentzel-Bardeen singularity is approached. This in turn triggers a simple and efficient mechanism to suppress antiferromagnetic fluctuations and to drive the system via a normal metallic state towards a superconducting phase.
\end{abstract}

PACS Numbers: 72.10.-d; 05.30.Fk;73.20.Dx

Recently there has been a renewed interest in onedimensional (1D) interacting fermion systems, both in the context of high temperature superconductivity [1], and in the context of mesoscopic systems [2 [5. 1D models are easier to handle than their 2D and $3 \mathrm{D}$ counterparts, and can yield useful information for the latter systems. It has long been recognized [6] that in 1D electron systems the low-energy long wave-length excitations around the Fermi surface have bosonic properties. The electron system is then represented as an harmonic fluid [7] 9], called Luttinger liquid [10], where the Fermi operators are expressed in terms of scalar Bose fields.

In this letter, we study the properties of a 1D system of correlated electrons, interacting with a short range repulsive potential, and coupled to small-momentum transfer bosons with acoustic dispersion. We concentrate first on acoustic phonons, and later on discuss an application to electron waveguides. While the low dimensionality and the short range forces rule out the possibility of long range order at any finite temperature $\mathrm{T}$, one can study which type of ordering fluctuations dominates the phase at vanishing $\mathrm{T}[\mathrm{B}]$ - charge/spin density waves (CDW/SDW) or singlet/triplet superconducting (SS/TS) fluctuations - and plot the equivalent of a phase diagram.

1D electron systems coupled to small-momentum phonons were considered more than four decades ago by Wentzel [11] and by Bardeen [12 as candidates for the theory of conventional superconductivity. It was pointed out that for a critical phonon coupling constant, the system becomes unstable, and the specific heat diverges as one approaches this critical point 13]: the retarded interaction mediated by the phonons induces a collapse of the system. Below, we refer to this singular point as the Wentzel-Bardeen (WB) singularity. Recently, an exactly solvable model similar to that of Ref. [13] was studied [14], with the conclusion that these small-momentum phonons can be ignored in typical metals since their effect is of the order $c^{2} / v_{F}^{2} \ll 1\left(v_{F}\right.$ is the Fermi velocity and $c$ the sound velocity). Consequently, the interest 15 17] shifted towards larger-momentum $\left(2 k_{F}\right)$ processes, where an electron is scattered by a phonon across the Fermi surface.

However, we shall show that the small-momentum phonons do play an important role in strongly correlated systems, as it is the velocity $u_{\rho}$ of particle-hole excitations which determines the "adiabatic parameter", $c^{2} / u_{\rho}^{2}$, and not the Fermi velocity $v_{F}$. In particular, we argue in the following that: 1) the coupling to smallmomentum transfer modes (such as acoustic phonons) is a non-perturbative effect as one reaches the WB singularity. 2) For the Hubbard model, the WB singularity can be reached for arbitrary electron-phonon coupling constant by increasing the filling factor towards half filling. 3) Near this WB singularity, CDW and SDW fluctuations are suppressed, and the system is pushed into a metallic and finally superconducting phase by slightly increasing the filling factor. Migdal's theorem does therefore not apply in the presence of strong correlations. Finally, it is worth pointing out that this high sensitivity towards doping is reminiscent of high temperature superconductivity materials.

We use the Luttinger liquid description generalized to electrons with spin [8], later on to be further specified to a Hubbard model [19]. Our starting point is the Hamiltonian, $H=H_{e}+H_{p h}+H_{e-p h}$, where

$$
H_{p h}=\frac{1}{2} \int d x\left[\zeta^{-1} \Pi_{d}^{2}+\zeta c^{2}\left(\partial_{x} d\right)^{2}\right]
$$

describes the free phonons, with $d$ the displacement field, $\Pi_{d}$ its canonical conjugate and $\zeta$ the mass density. The electron-phonon coupling is [20]: 


$$
H_{e l-p h}=g \sqrt{\frac{\pi}{2}} \sum_{s} \int d x \psi_{s}^{\dagger} \psi_{s} \partial_{x} d
$$

where $g$ is the coupling constant, and $\psi_{s}=$ $e^{i k_{F} x} \psi_{s+}+e^{-i k_{F} x} \psi_{s-}$ is the electron field operator. In terms of boson fields, $\psi_{s \pm}(\mathbf{x}) \propto \exp \left[i \sqrt{\pi}\left( \pm \varphi_{s}(\mathbf{x})-\right.\right.$ $\left.\left.\left.\int^{x} \Pi_{s}\left(x^{\prime}, \tau\right) d x^{\prime}\right)\right)\right]$ (here $\mathbf{x}=(x, \tau)$ ) representing right and left moving electrons. The electron density in Eq. (0.2) reduces to $\sum_{s} \psi_{s}^{\dagger} \psi_{s}=\sqrt{2 / \pi} \partial_{x} \varphi_{\rho}$, with $\varphi_{\rho, \sigma}=$ $\left(\varphi_{\uparrow} \pm \varphi_{\downarrow}\right) / \sqrt{2}$ the charge and spin fields (similarly for the canonical conjugate fields $\Pi_{\rho, \sigma}$ ). We have neglected the fast oscillating terms in the density which give rise to $2 k_{F}$ phonon induced backscattering. The electronic Hamiltonian is $H_{e l}=H_{\rho}+H_{\sigma}$, with

$$
H_{\nu}=\frac{1}{2} \int d x\left[u_{\nu} K_{\nu} \Pi_{\nu}^{2}+\frac{u_{\nu}}{K_{\nu}}\left(\partial_{x} \varphi_{\nu}\right)^{2}\right],
$$

with $\nu=\rho, \sigma$. The phonons couple only to the charge degrees of freedom, and this property is preserved in the propagators calculated below. In Eq. (0.3), the electron interaction is included in the parameters $K_{\rho}, K_{\sigma}$, and the charge (spin) velocities $u_{\rho}\left(u_{\sigma}\right)$. This model belongs to the same universality class as the multicomponent Tomonaga-Luttinger model [18].

To calculate Green functions, we use a Lagrangian formulation, where the partition function is represented as a functional integral. As we will be calculating Green functions which involve fermion operators only, the phonon degrees of freedom are integrated out right away. This leaves us with an effective action for the charge degrees of freedom, which reads in Fourier representation: $S_{\rho}=(2 \beta L)^{-1} \sum_{\mathbf{k}} D_{\rho}(\mathbf{k})\left|\varphi_{\rho}(\mathbf{k})\right|^{2}(\mathbf{k}=(k, \omega))$, with the inverse propagator:

$$
D_{\rho}(\mathbf{k})=\frac{1}{K_{\rho} u_{\rho}}\left(\omega^{2}+u_{\rho}^{2} k^{2}-\frac{b^{2} k^{4}}{\omega^{2}+c^{2} k^{2}}\right),
$$

where $b=g \sqrt{K_{\rho} u_{\rho} / \zeta}$. The first two terms of Eq. (0.4) represent the contribution of the free charge field, and the retarded, attractive coupling associated with the phonons appears in the third term. At the WB point the charge density propagator, $k^{2} / D_{\rho}(\mathbf{k})$, becomes proportional to $\omega^{-2}$ and signals an instability towards long wave-length density fluctuations.

We first calculate the single-particle Green function:

$$
G_{s}(x, \tau)=-<T \psi_{s}(x, \tau) \psi_{s}^{\dagger}(0,0)>,
$$

where $T$ is the imaginary time ordering operator. This quantity has been calculated before [21] (by diagrammatic methods) for spinless fermions coupled to acoustic phonons, but in the perturbative limit $c^{2} / v_{F}^{2} \ll 1$, where the WB singularity is absent. Using the decomposition of the Fermi operators into right and left moving components, and after normal ordering [3,22], the calculation of the Green function is reduced to an evaluation of Gaussian integrals. The result is ( $a$ is the lattice constant):

$$
\begin{aligned}
G_{s}(x, \tau)= & \frac{2 \pi}{a}\left|\frac{a}{x+i u_{\sigma} \tau}\right|^{K_{\sigma} / 4+1 / 4 K_{\sigma}} \\
& \times \prod_{\beta= \pm}\left|\frac{a}{x+i v_{\beta} \tau}\right|^{K_{\rho} u_{\rho} F_{\beta} / 4 v_{\beta}+C_{\beta} / 4 K_{\rho}} \\
& \times \sum_{\alpha= \pm} e^{i \alpha k_{F} x}\left(\frac{s \alpha x+i u_{\sigma}|\tau|}{i\left|x+i u_{\sigma} \tau\right|}\right)^{\operatorname{sgn}(\tau) / 2} \\
& \times \prod_{\gamma= \pm}\left(\frac{\alpha x+i v_{\gamma}|\tau|}{i\left|x+i v_{\gamma} \tau\right|}\right)^{\operatorname{sgn}(\tau) F_{\gamma} / 2} \\
& \times n_{\alpha}(x, \tau),
\end{aligned}
$$

with the normal ordering contribution:

$$
n_{\alpha}(x, \tau)=\operatorname{sgn}(\tau) \prod_{\nu=\rho, \sigma} e^{-\alpha \pi \operatorname{sgn}(\tau)\left[\theta_{\nu}(x, \tau), \varphi_{\nu}(0,0)\right] / 2},
$$

and thus $\left|n_{\alpha}\right|=1$. If $x / L \neq 0$ and $\tau$ arbitrary, $n_{\alpha}(x, \tau)=$ $\operatorname{sgn}(\tau) \exp (i \alpha \pi x \operatorname{sgn}(\tau) / L)$, and if $\tau=0^{ \pm}$and $x$ arbitrary, $n_{\alpha}\left(x, 0^{ \pm}\right)= \pm i \alpha|x+i \alpha a| /(x+i \alpha a)$. Near $\mathbf{x}=0$ one has to add $a$ to $v|\tau|$ in Eq. (0.6). We introduced the velocities, $v_{ \pm}^{2}=\left[u_{\rho}^{2}+c^{2} \mp \sqrt{\left(u_{\rho}^{2}-c^{2}\right)^{2}+4 b^{2}}\right] / 2$, for the electronic collective excitations. These velocities characterize the roots of the propagator (0.4), and arise from the hybridization of the charge and phonon excitations. Note that it is the charge velocity $u_{\rho}$ (not $v_{F}$ ) which appears here. The exponents are given by $C_{ \pm}=u_{\rho}\left(v_{ \pm}^{2}-c^{2}+b^{2} / u_{\rho}^{2}\right) /\left[\left(v_{ \pm}^{2}-v_{\mp}^{2}\right) v_{ \pm}\right]$, and $F_{ \pm}=$ $\left(v_{ \pm}^{2}-c^{2}\right) /\left(v_{ \pm}^{2}-v_{\mp}^{2}\right)$.

At large distances, the Green function decays as a power law, $G_{s}(x, 0) \propto|x|^{-1-\delta}$, with $\delta=K_{\sigma} / 4+$ $1 /\left(4 K_{\sigma}\right)-1+B /\left(4 K_{\rho}\right)+A K_{\rho} / 4$. The electron-phonon parameters are defined by $A=u_{\rho}\left(1+c^{2} / v_{+} v_{-}\right) /\left(v_{+}+\right.$ $\left.v_{-}\right) \geq 1$, and $B=u_{\rho}\left(1+v_{+} v_{-} / u_{\rho}^{2}\right) /\left(v_{+}+v_{-}\right) \leq 1$. These parameters play a crucial role in the discussion of the ordering fluctuations below. In the limit $g \rightarrow 0$, $A=B=1$. Let us now consider the case $g \neq 0$, where $A>1$ and $B<1$ 222]: As $u_{\rho} / K_{\rho}$ approaches the critical value $u_{\rho}^{*} / K_{\rho}^{*}=g^{2} /\left(\zeta c^{2}\right)$ from above, $v_{+}^{2}$ tends to zero, and $v_{-}^{2}$ to $u_{\rho}^{* 2}+c^{2}$. As a result, the exponent $A$ diverges and $B$ decreases to the finite value $B^{*}=u_{\rho}^{*} / \sqrt{u_{\rho}^{* 2}+c^{2}}<1$. For $u_{\rho} / K_{\rho}<u_{\rho}^{*} / K_{\rho}^{*}$ the velocity $v_{+}$becomes complex and the model becomes unphysical. Thus we must require that $u_{\rho} / K_{\rho} \geq u_{\rho}^{*} / K_{\rho}^{*}$, or equivalently that $b /\left(c u_{\rho}\right) \leq 1$, the equality sign defines the WB singularity [23]. This singular behavior is a non-perturbative effect of the electron-phonon coupling 24] and originates from the instability of the propagator (0.4). Because $\delta>0$, if $g \neq 0$ [22], the Green function decays faster than $1 / x$, the free electron result. From (0.6), we find the momentum distribution function near $k_{F}, N(k) \simeq N\left(k_{F}\right)-\kappa \operatorname{sgn}\left(k-k_{F}\right)\left|k-k_{F}\right|^{\delta}$, with $\kappa$ some constant of order one, and $N\left(k_{F}\right)=\Gamma(1 / 2+$ $\delta / 2) /[2 \sqrt{\pi} \Gamma(1+\delta / 2)]$. This continuous but non-analytic 
behavior at the Fermi surface is characteristic for Luttinger liquids [6] 8]. The presence of phonons induces this Luttinger liquid behavior even without electron-electron interaction $\left(K_{\rho}=K_{\sigma}=1\right)$.

We now turn to the discussion of the ordering fluctuations characterized by two-particle response functions. The definitions for $N(\mathbf{x}), \chi(\mathbf{x}), \Delta_{s}(\mathbf{x})$ and $\Delta_{t}(\mathbf{x})$ which describe CDW, SDW, singlet (SS) and triplet (TS) superconducting fluctuations are given in 8,9,9]. The calculation of these Green functions will be given elsewhere 22. Here, we give the final result in Matsubara representation:

$$
\begin{aligned}
& N(x, \tau) \propto \prod_{\beta= \pm}\left|x+i v_{\beta} \tau\right|^{-u_{\rho} K_{\rho} F_{\beta} / v_{\beta}}\left|x+i u_{\sigma} \tau\right|^{-K_{\sigma}} \\
& \chi(x, \tau) \propto \prod_{\beta= \pm}\left|x+i v_{\beta} \tau\right|^{-u_{\rho} K_{\rho} F_{\beta} / v_{\beta}}\left|x+i u_{\sigma} \tau\right|^{-1 / K_{\sigma}} \\
& \Delta_{s}(x, \tau) \propto \prod_{\beta= \pm}\left|x+i v_{\beta} \tau\right|^{-C_{\beta} / K_{\rho}}\left|x+i u_{\sigma} \tau\right|^{-K_{\sigma}} \\
& \Delta_{t}(x, \tau) \propto \prod_{\beta= \pm}\left|x+i v_{\beta} \tau\right|^{-C_{\beta} / K_{\rho}}\left|x+i u_{\sigma} \tau\right|^{-1 / K_{\sigma}}
\end{aligned}
$$

The signature for a particular ordering fluctuation to be present is given by the divergence of the Fourier transform of the corresponding response function at low frequency and small momentum relative to $q=2 k_{F}(q=0)$ for $N$ and $\chi\left(\Delta_{s, t}\right)$. We thus obtain the following criteria 22]:

$$
\begin{aligned}
K_{\rho} A+K_{\sigma} \leq 2 & \\
K_{\rho} A+1 / K_{\sigma} \leq 2 & (\mathrm{CDW}) \\
B / K_{\rho}+K_{\sigma} \leq 2 & (\mathrm{SS}) \\
B / K_{\rho}+1 / K_{\sigma} \leq 2 . & (\mathrm{TS})
\end{aligned}
$$

Since $A>1$ and $B<1$ for $g \neq 0$, we see that the Cooper instability is always present for non-interacting electrons coupled to phonons. The exponents can in principle be recovered in [18], which treats formally an arbitrary number of coupled charge fields: here we have the advantage of having explicit analytic expressions.

We now specialize to a Hubbard model for the correlated electrons. In this case 19], $K_{\sigma}=1$, and the CDW and the SDW (the SS and the TS) response functions have apparently the same exponents (except at half filling). However, logarithmic corrections due to marginally irrelevant operators in the spin channel favor SDW (TS) over CDW (SS) fluctuations [25]. The remaining parameters $u_{\rho}$ and $K_{\rho}$ of the Luttinger liquid Hamiltonian are obtained for arbitrary on-site repulsion $U$ and filling factor $n$ by solving two integral equations [1922] describing the ground state properties [26] and the spectrum of charge excitations 27.

Our results are plotted in Fig. 1 a) and b): for fixed $U / t$ (Fig. 11 a), where $t$ is the bandwidth, we determine which fluctuations dominate as a function of $n$ and an effective electron-phonon parameter $b /\left(u_{\rho} c\right)$ : for convenience we consider in these plots $n(U)$ and $b /\left(u_{\rho} c\right)$ as independent parameters. For small $b /\left(u_{\rho} c\right)$, SDW (i.e. antiferromagnetic) ordering fluctuations dominate for arbitrary filling factor. As this parameter is increased, we reach (away from half filling) a region for which no response function diverges: we refer to this "phase" as the metallic region. At low $U$ and $b /\left(u_{\rho} c\right) \ll 1$ analytical results confirm the existence of this intermediate phase [22]. By further increasing $b /\left(u_{\rho} c\right)$, the region where superconducting fluctuations dominate is reached. On the other hand, near half filling, the correlation effects suppress the superconducting phase. (For larger values of $U$, the superconducting region shrinks towards the region where $b /\left(u_{\rho} c\right)=1$, and the SDW region grows as expected [22].) Next, we choose quarter filling $(n=1 / 2)$, and plot in Fig. 11 b) the phase diagram as a function of $U$ and the phonon coupling. At low $U, K_{\rho} \approx 1$, and the system is superconducting because of the Cooper instability. As $U$ is further increased, one crosses the metallic and the SDW (CDW) phase, because the phonon-mediated attractive interaction is overcome by the instantaneous repulsion between electrons.

In both Figs. 1 a) and b), the upper line $b /\left(u_{\rho} c\right)=1$ corresponds to the WB singularity. We now discuss the relevance of this singularity for the Hubbard model. Plotting $u_{\rho} / K_{\rho}$ for several values of $U$ as a function of the filling factor (Fig. 2), we notice the well-known fact [19] that $u_{\rho} / K_{\rho}$ vanishes as one approaches half filling (and, of course, zero filling). Thus, the WB singularity at $u_{\rho}^{*} / K_{\rho}^{*}$ can be reached for arbitrary values of the coupling constant $g(\neq 0)$. Near half filling, as one approaches $u_{\rho}^{*} / K_{\rho}^{*}$ from lower filling factors, the divergence in $A$ triggers a dramatic suppression of the SDW (CDW) fluctuations, and the system is driven into the metallic phase [28]. Moreover, since $B \rightarrow B^{*}$ as $u_{\rho} \rightarrow u_{\rho}^{*}$, and $B^{*} \propto g^{2}$, the SS/TS condition $B \leq K_{\rho}$ can be met near half filling for sufficiently small $g \neq 0$ (since $\left.1 / 2 \leq K_{\rho} \leq 1\right)$. Hence, the system can finally be driven into the superconducting phase by approaching half filling. In summary, the conjunction of the coupling to small momentum acoustic modes with the reduction of the charge velocity near half filling provides a very efficient mechanism to suppress SDW (CDW) order, and to drive the system into a metallic and finally superconducting phase. This mechanism need not be limited to the 1D Hubbard model, but could occur in other situations where strong correlations play an important role.

We conclude with an application to mesoscopic quasi$1 \mathrm{D}$ wires. The phonon field in Eqs.(0.1) and (0.2) now represents the charge excitations of another transverse state. This model was used in [5] to describe the transport properties of a multimode wire [29]. The Fermi velocities associated with the two modes are comparable and therefore the retarded attractive interaction is much stronger than for phonons. Beyond a critical coupling, superconducting fluctuations are induced first in 
the branch with the lower Fermi velocity, and then in both modes. This could be probed e.g. by studying the periodicity of the persistent current of a two channel mesoscopic ring in a heterostructure [30], as a function of electron density. The flux causes a twist in the boundary conditions, but the bulk properties are unaffected. The mutual inductance of the two modes is negligible because of the small ratio $\alpha v_{F} / c_{l}\left(\alpha \sim 137\right.$ and $c_{l}$ is the velocity of light (4)). In the metallic regime, the power spectrum has a peak associated with the flux quantum $\phi_{0}=h c / e$, whereas in the superconducting case, this peak should give place to a peak at the superconducting flux quantum $\phi_{0} / 2$

\section{ACKNOWLEDGMENTS}

We thank A. J. Leggett, C.P. Enz, and S. Trugman for useful discussions. The work of D.L. is supported by NSERC of Canada.

* Permanent address as of October 1994: Institut Laue Langevin, B.P. 156, 38042 Grenoble, France.

[1] P. W. Anderson, Phys. Rev. Lett. 67, 3844 (1991).

[2] C. L. Kane and M. P. A. Fisher, Phys. Rev. Lett. 68, 1220 (1992).

[3] D. Loss, Phys. Rev. Lett. 69, 4630 (1992).

[4] D. Loss and Th. Martin, Phys. Rev. B. 47, 4619 (1993).

[5] K. A. Matveev and L. I. Glazman, Phys. Rev. Lett. 70, 990 (1993).

[6] D. C. Mattis and E. H. Lieb, J. Math. Phys. 6, 304 (1965).

[7] A. Luther and I. Peschel, Phys. Rev. B 9, 2911 (1974).

[8] V. J. Emery, in Highly Conducting One Dimensional Solids, J. T. Devreese, R. P. Evrard and V. E. van Doren eds. (Plenum, New York, 1979), p.327.

[9] J. Sólyom, Adv. Phys. 28, 201 (1979).

[10] F. D. M. Haldane, J. Phys. C 14, 2585 (1981); Phys. Rev. Lett. 47, 1840 (1981).

[11] G. Wentzel, Phys. Rev. 83, 168 (1951).

[12] J. Bardeen, Rev. Mod. Phys. 23, 261 (1951).

[13] S. Engelsberg and B. B. Varga Phys.Rev. 136, A1582 (1964).

[14] J. Voit and H. J. Schulz, Mol. Cryst. Liq. Cryst. 119, 449 (1985).

[15] J. Voit and H. J. Schulz, Phys. Rev. B 34, 7429 (1986); 36, 968 (1987); 37, 10068 (1988).

[16] G. T. Zimanyi, S. A. Kivelson and A. Luther, Phys. Rev. Lett. 60, 2089 (1988).

[17] J. Voit, Phys. Rev. Lett. 64, 323 (1990).

[18] K. Penc and J. Sólyom, Phys. Rev. B 47, 6273 (1993).

[19] H. J. Schulz, Phys. Rev. Lett. 64, 2831 (1990); Int. J. of Mod. Phys. B 5, 57 (1991).
[20] A. Fetter and D. Walecka, Many Particle Physics (McGraw-Hill, New York, 1969).

[21] M. Apostol and I. Baldea, Phys. Lett. 88A, 73 (1982); J. Phys. C 15, 3319 (1982).

[22] Th. Martin and D. Loss, preprint (1993).

[23] This singularity was left unnoticed in Ref. [14 (only forward scattering is includede there), and the exponents given there seem to be incorrect.

[24] Note also that the limits $g \rightarrow 0$ and $u_{\rho} \rightarrow u_{\rho}^{*}$ do not commute.

[25] T. Giamarchi and H.J. Schulz, Phys. Rev. B 39, 4620 (1989).

[26] E. H. Lieb and F. Y. Wu, Phys. Rev. Lett. 20, 1445 (1968).

[27] C. F. Coll, Phys. Rev. B 9,2150 (1974).

[28] Note that the model becomes unphysical when we go below the WB singularity $u_{\rho}^{*} / K_{\rho}^{*}$ in Fig. 2 by increasing (lowering) the filling factor towards half (zero) filling.

[29] For further properties of coupled chains see also M. Fabrizio, A. Parola and E. Tosatti, Phys. Rev. B 46, 3156 (1992); A.M. Finkelstein and A.I. Larkin, Phys. Rev. B47, 10461 (1993).

[30] D. Mailly, C. Chapelier, and A. Benoit, Phys. Rev. Lett. 70, 2020 (1993).

FIG. 1. a) Phase diagram of the Hubbard model coupled to phonons, as a function of filling factor and phonon coupling constant $b / c u_{\rho}$, for the choices $U / t=0.3$ and $c=a t$ (this corresponds e.g.to $\left(c / u_{\rho}\right)^{2} \approx 1 / 2$ at quarter filling $(n=1 / 2)$. $S D W, M$, and $S C$ label the SDW, intermediate, and (triplet) superconducting regions. b) Phase diagram of the Hubbard model coupled to phonons at quarter filling $(n=1 / 2)$, as a function of $U / t$ and $b / c u_{\rho}$.

FIG. 2. Plot of $u_{\rho} / K_{\rho}$ as a function of the filling factor $n$. Note the abrupt change for small values of $U$ as one approaches half filling. 